\title{
Metastable States in Cellular Automata for Traffic Flow
}

\author{
R. Barlovic ${ }^{1}$, L. Santen ${ }^{2}$, A. Schadschneider ${ }^{2}$ and M. \\ Schreckenberg ${ }^{1}$
}

1 Theoretische Physik/FB 10

Gerhard-Mercator-Universität Duisburg

D-47048 Duisburg, Germany

email: barlovic,schreck@uni-duisburg.de

${ }^{2}$ Institut für Theoretische Physik

Universität zu Köln

D-50937 Köln, Germany

email: santen, as@thp.uni-koeln.de

August 13, 2018

Dedicated to J. Zittartz on the occasion of his 60th birthday

\begin{abstract}
Measurements on real traffic have revealed the existence of metastable states with very high flow. Such states have not been observed in the Nagel-Schreckenberg (NaSch) model which is the basic cellular automaton for the description of traffic. Here we propose a simple generalization of the NaSch model by introducing a velocitydependent randomization. We investigate a special case which belongs to the socalled slow-to-start rules. It is shown that this model exhibits metastable states, thus sheding some light on the prerequisites for the occurance of hysteresis effects in the flow-density relation.
\end{abstract}




\section{Introduction}

A few years ago, Nagel and Schreckenberg [1] (NaSch) have proposed a probabilistic cellular automaton (CA) for the description of single-lane highway trafficf. Using very simple rules, this model is able to reproduce the basic phenomena encountered in real traffic, e.g. the occurance of phantom traffic jams ('jams out of nowhere'). The NaSch model is 'minimal' in the sense that every simplification of the rules no longer produces realistic results. On the other hand, for the description of more complex situations (e.g. multi-lane traffic, ramps)f or for a proper modelling of the 'fine-structure' of traffic flow, additional rules have to be added and/or the basic rules have to be modified.

The NaSch model [1] is a probabilistic cellular automaton. Space and time (and hence the velocities) are discrete. The road is divided into cells of length $7.5 \mathrm{~m}$. Each cell can either be empty or occupied by just one car. The state of car $j(j=1, \ldots, N)$ is characterised by its momentary velocity $v_{j}\left(v_{j}=0,1, \ldots, v_{\max }\right)$. The state of the system at time $t+1$ can be obtained from the state at time $t$ by applying the following four rules to all cars at the same time (parallel dynamics):

R1: Acceleration: $v_{j}(t) \rightarrow v_{j}\left(t+\frac{1}{3}\right)=\min \left\{v_{j}(t)+1, v_{\max }\right\}$

R2: Braking: if $v_{j}\left(t+\frac{1}{3}\right)>d_{j}(t)$ then $v_{j}\left(t+\frac{2}{3}\right)=d_{j}(t)$ else $\quad v_{j}\left(t+\frac{2}{3}\right)=v_{j}\left(t+\frac{1}{3}\right)$

R3: Randomization: $v_{j}\left(t+\frac{2}{3}\right) \stackrel{p}{\rightarrow} v_{j}(t+1)=\max \left\{0, v_{j}\left(t+\frac{2}{3}\right)-1\right\}$ with probability $p$

R4: Driving: car $j$ moves $v_{j}(t+1)$ cells.

Here $d_{j}(t)$ denotes the number of empty cells in front of car $j$, i.e. the gap or headway. One timestep $t \rightarrow t+1$ corresponds to approximately $1 \mathrm{sec}$ in real time [1].

In the spirit of modelling complex phenomena in statistical physics, the NaSch model does not try to describe traffic flow very accurately on a microscopic level. Macroscopic effects observed in real traffic, e.g. the spontaneous formation of jams, can be understood by introducing just one simple stochastic parameter, the braking probability $p$. Note that the motion of a single car might exhibit (on short timescales) unrealistic features, like stopping from maximum velocity within a few timesteps without any reason. However, after averaging over the motion of all cars or on long timescales, the NaSch model produces quite realistic results. Therefore one should not try to relate these large fluctuations to those observed in real traffic.

Besides the CA models which are discrete in space and time, several other approaches to traffic flow have been discussed recently. Among these are space-continuous mod-

\footnotetext{
${ }^{1}$ For an overview of other approaches, see e.g. [2, 3, 4, 胿]

${ }^{2}$ For applications to urban traffic, see e.g. [6, 6].
} 
els in discrete time like the model of Krauss et. al. [8, 9], as well as models continuous both in space and time, e.g. the macroscopic (fluid-dynamical) models [10], the optimum-velocity model [11], coupled-map models [12] and gas-kinetic models [13]. For further references we refer to [2, 3, , 4.

In the present paper we want to investigate hysteresis effects encountered in empirical observations [14, 15, 16]. Such effects are related to the existence of metastable states in certain density regimes. In the NaSch model these states have not be observed. Here we want to present slightly modified models which are able to produce metastable states and hysteresis. As a consequence, the occurance of metastable states is therefore not related to the use of realistic braking rules, a continuum description or deterministic models.

In order to establish the existence of metastable states one can follow two basic strategies. In the first method, the density of cars is changed adiabatically by adding or removing vehicles from the stationary state at a certain density. Starting in the jamming phase (large densities) and removing cars, one obtains the lower branch of the hysteresis curve. On the other hand, by adding cars to a free flowing state (low densities), one obtains the upper branch. The second method does not require changing the density. Instead one starts from two different initial conditions, the megajam and the homogeneous state. The megajam consists of one large, compact cluster of standing cars. In the homogeneous state, cars are distributed equidistantly (with one large gap for incommensurate densities).

In certain density regimes the fundamental diagram can consist of two branches. In the upper branch (with higher flow) there are almost no interactions between the cars and the system remains in a homogeneous, jam-free state. In the lower branch, however, the system is in a 'phase-separated' state, consisting of one large jam and a free-flowing part.

Experimental observations [17] suggest that a reduction of the outflow from a jam compared to the maximum possible flow 3 is an important ingredient for the occurrance of metastable states with large lifetimes. Such a reduction can be implemented by so-called slow-to-start rules, where standing cars accelerate with lower probability than moving cars. It leads to a downstream flow with a relatively low density corresponding to the lower branch of the hysteresis curve. Due to the reduction of the density in the outflow region of a jam, the flow in the jammed state is significantly lower than the flow of the homogeneous state at the same density.

The paper is organized as follows: In Sect. 2 two models with slow-to-start rules are investigated. In Sect. 3 we introduce a slightly modified NaSch model with a velocity-dependent randomization. In this way, one incorporates the basic ingredients necessary to produce hysteresis. In the concluding Section 1 we summarize our results and compare with those for other models and real traffic.

\footnotetext{
${ }^{3}$ This reduction is often referred to as 'capacity drop'.
} 


\section{Models with slow-to-start rules}

In this Section we briefly present results for two models with slow-to-start (s2s) rules [18], the $\mathrm{T}^{2}$ model [19, 20] and the BJH model [21]. These models have been introduced in order to model the restart behaviour of stopped cars in a more realistic fashion. As will be demonstrated below, these s2s rules are an important ingredient for the occurance of metastable states, although the authors of [19, 21] did not realize the connection between s2s rules and metastability encountered in real traffic.

\section{$2.1 \quad \mathrm{~T}^{2}$ model}

Takayasu and Takayasu $\left(\mathrm{T}^{2}\right)$ [19 have first suggested a CA model with a s2s rule. This rule has been generalized in 18 as follows: A standing car with exactly one empty cell in front of it accelerates with probability $q_{t}=1-p_{t}$, while all other cars accelerate deterministically. The other update rules (R2-R4) of the NaSch model are unchanged, e.g. all cars are still subject to the randomization step. Due to this modification already for $v_{\max }=1$ the particle-hole symmetry is broken.

In Fig. 1 1 we show the fundamental diagram of the $\mathrm{T}^{2}$ model with $v_{\max }=5, p=0.01$ and $p_{t}=0.75$. The system size used for the simulation was $L=1000$. In order to equilibrate the system, 10000 lattice updates have been performed. The data shown in Fig. 1 represent an average over 100000 sweeps through the lattice.

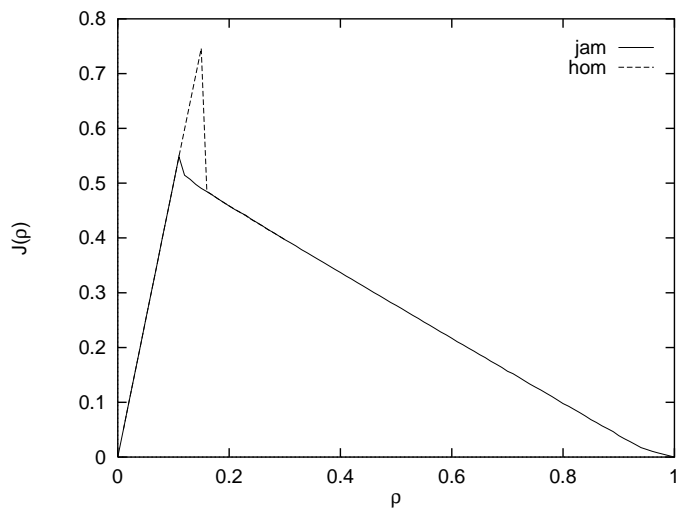

Figure 1: Fundamental diagram of the $\mathrm{T}^{2}$ model $\left(v_{\max }=5, p=0.01, p_{t}=0.75\right)$ obtained by starting from two different initial conditions, a completely jammed state (full line) and a homogeneous state (broken line). The peak of the full line is a finite-size effect.

Comparing the simulation results with those for the NaSch model, two qualitative differences have to be discussed. First, the fundamental diagram has an inflection point in the high density regime and second the flow behaviour is non-unique in a density regime below the density of maximum flow $\rho_{\max }$. 
The existence of an inflection point for large $p_{t}$ has first been noticed in $18 \|$ for the case $v_{\max }=1$. In contrast, the fundamental diagram of the NaSch model is always convex. The different behaviour of the $\mathrm{T}^{2}$ model for large $p_{t}$ and large densities $\rho$ is related to the fact that space is not used as efficiently as in the NaSch model. One finds a similar behaviour in space-continuous models [22]. There is some experimental evidence that in certain situations the shape of the fundamental diagram differs from the convex form. This behaviour of the average flow can be easily obtained tuning the parameter $p_{t}[18,23]$.

The non-unique behaviour of the flow for densities just below $\rho_{\max }$ is due to the fact that for these densities the average flow still depends on the initial configuration. The measurements in Fig. 1 have been performed by applying the second method described in the introduction. The lower branch of the fundamental diagram corresponds to measurements starting from a initially completely jammed configuration while the upper branch has been obtained starting with a homogeneous initialization. It should be mentioned that the same result can also be obtained by applying the first method, i.e. by changing the density adiabatically. Since the flow depends on the history of the system, diagrams like those of Fig. 1 are usually called hysteresis curves.

For low densities the stationary state consists of homogeneous configurations which are completely jam-free. At higher values of the global density the configurations contain one large jam. In contrast to the NaSch model, no spontaneous formation of jams in the outflow region of the large jam has been observed for the system sizes we took into account. Therefore the jammed states are phase separated states, unlike for the case of the NaSch model.

It should be noted that the maximum value of the average flow still depends on the number of updates as well as on the system size. Therefore we can not exclude that the stationary value of the average flow is unique in the thermodynamic limit. Nevertherless the metastable states are extremely stable, even for large systems. Moreover, in contrast to standard problems of statistical mechanics, not the thermodynamic limit is relevant for practical purposes, but the behavior of systems of finite length (note that 10000 lattice sites correspond to a road of length $75 \mathrm{~km}$ in reality). Since in reality roads and observation times are always finite, the above results are sufficient for all practical purposes.

In Fig. 2 we show the fundamental diagram for $v_{\max }=1, p=0.5$ and $p_{t}=1$, i.e. stopped cars can only move if there are at least two empty cells in front. Obviously completely blocked states exist for densities $\rho \geq 0.5$, where the number of empty cells in front of each car is smaller than two. Since fluctuations are absent in those states, they have an infinite lifetime. Therefore the flow in the stationary state is zero. In the region $0.5 \leq \rho \lesssim 0.66$ states with a finite flow exist. Although these states are not stationary, one has to perform an extremely large number of update steps until the flow vanishes for large system sizes and densities slightly above $\rho=0.5$, because the number of blocked configurations is very small compared to the total number of 
configurations. Precisely at $\rho=0.5$, the blocked state is unique and the typical time to reach this state diverges exponentially with the system size. Therefore we used very small systems in order to obtain the lower branch of the fundamental diagram.



Figure 2: Fundamental diagram of the $\mathrm{T}^{2}$ model $\left(v_{\max }=1, p=0.5, p_{t}=1\right)$ for two system sizes. For densities slightly above $\rho=0.5$ the stationary state could only be reached for the smaller system.

Note that the mechanism for metastability in the case $p_{t}=1$ is different. The hysteresis curve in Fig. 2 has been obtained by starting from two different homogeneous states, differing only in the velocity of the cars. The jammed branch is reached by starting with a configuration where all cars $j$ have a velocity $v_{j}(t=0)=0$, whereas the upper branch corresponds to an initialization $v_{j}(t=0)=v_{\max }$. In contrast, by following the first method described in the introduction, one would not find hysteresis, since a megajam initialization would also yield the high-current branch.

The reason for the occurance of hysteresis in the limit $v_{\max }=1$ and $p_{t}=1$ is the existence of a "geometrical" phase transition. If the critical density is exceeded, the cars can no longer move since there is not enough free space. The "geometrical" phase transition makes it possible to find hysteresis even in the case $v_{\max }=1$.

Finally we want to remark that the $p_{t}=1$ limit of the $\mathrm{T}^{2}$ model is some sense complementary to the cruise-control limit [24] of the NaSch model. In the $\mathrm{T}^{2}$ model the completely blocked state is stabilized due to the absence of fluctuations whereas in the cruise-control limit one finds the absence of fluctuations for homogeneous states at low densities.

\subsection{BJH model}

The s2s rule of the $\mathrm{T}^{2}$ model is a 'spatial' rule. The range of interaction for standing cars is larger than in the NaSch model and the restart behaviour depends only on the spatial arrangement of the vehicles. However, there are other ways of implementing a s2s behaviour. In the Benjamin-Johnson-Hui (BJH) model [21] cars which had 
to brake due to the next car ahead, will move on the next opportunity only with probability $1-p_{s}$. Note that in contrast to the $\mathrm{T}^{2}$ this slow-to-start rule requires 'memory', i.e. it is a 'temporal' rule depending on the number of trials and not on the free space available in front of the car.

For the BJH model no metastable states and hysteresis effects have been found until now since only the case $v_{\max }=1$ has been investigated thoroughly [21, 18]. For higher velocities we expect the occurance of metastable states, since also in the BJH model the outflow from a jam is smaller than the maximal flow.

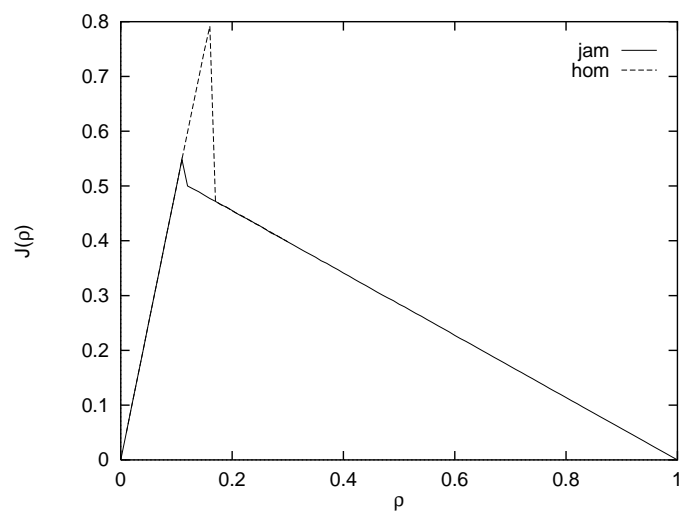

Figure 3: Fundamental diagram of the BJH model $\left(v_{\max }=5, p=0.01, p_{s}=0.75\right)$ obtained using two different initial conditions, namely a completely jammed state (full line) and a homogeneous state (broken line).

Our simulations show that for $v_{\max }>1$ the overall behaviour of the BJH model is very similar to that of the $\mathrm{T}^{2}$ model (for $p_{t}<1$ ). Therefore we are not going to discuss it here. In Fig. 3 we show a typical fundamental diagram. There is, however, no inflection point and the fundamental diagram is convex for all $p_{s}$. This supports the view that the existence of an inflection point is a 'spatial' effect.

\section{NaSch model with velocity-dependent random- ization}

Here we present a simple generalization of the NaSch model which incorporates slowto-start behaviour without introducing memory (like in the BJH model) or a longerranged interaction (like in the $\mathrm{T}^{2}$ model). This new s2s rule is therefore neither temporal nor spatial.

Instead, a velocity-dependent randomization (VDR) parameter $p=p(v(t))$ is introduced. This parameter has to be determined before the acceleration step R1. For 
simplicity we here study only the case

$$
p(v)= \begin{cases}p_{0} & v=0 \\ p & v>0\end{cases}
$$

which already contains the most important features of the general case [25]. Since we are interested in hysteresis phenomena, we restrict ourselves to the case $p_{0} \leq p$. Note that for $p_{0}=p$ the NaSch model is recovered. The cruise-control limit 24 corresponds to the choice $p\left(v_{\max }\right)=0$ and $p(v)=p$ for $v<v_{\max }$.

In the following we will use a maximum velocity $v_{\max }=5$, braking probability $p=1 / 64$ of the moving cars and a higher value $p_{0}=0.75$ for the braking probability of stopped cars. Simulation runs have been performed for periodic systems with $L=10000$ lattice sites.

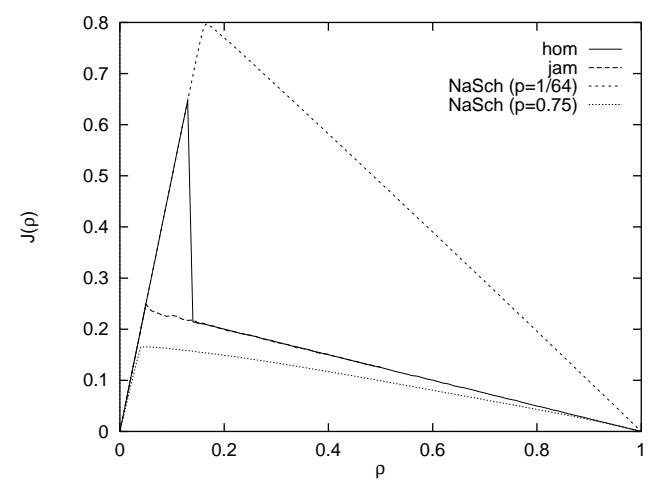

Figure 4: Fundamental diagram of VDR model $\left(v_{\max }=5, p_{0}=0.75, p=1 / 64\right.$, $L=10000)$. For comparison the fundamental diagrams of the NaSch model with $p=0.75$ and $p=1 / 64$ are given.

Fig. 4 shows the fundamental diagram of the modified model. Obviously the average flow $J(\rho)$ can take two values in the density interval between $\rho_{1}$ and $\rho_{2}$ depending on the chosen initialization. The larger values of the average flow can be obtained using a homogeneous initialization of the system. The lower branch is obtained starting from a completely jammed state. Moreover, varying the particle number adiabatically, one can trace a hysteresis loop. One gets the upper branch by adding cars to the stationary state with $\rho<\rho_{1}$ and the lower one by removing cars from the stationary state with $\rho>\rho_{2}$. For a fixed value of $p, \Delta J=J\left(\rho_{2}\right)-J\left(\rho_{1}\right)$ depends linearly on $p_{0}$ for wide a range of parameters.

Increasing the system size, we observe a decrease of the density $\rho_{2}$ towards the branching density $\rho_{1}$. The jammed states become stable for densities $\rho \geq \rho_{1}$ even for global densities very close to $\rho_{1}$. Again it should be noted that the homogeneous states have extremely long life-times and should therefore be relevant for realistic systems.

It is instructive to compare the fundamental diagram of the VDR model with those of the corresponding NaSch models. For small densities $\rho \ll 1$ there are no slow 
cars in the VDR model since interactions between cars are extremely rare. Here the flow is given by $J(\rho) \approx \rho\left(v_{\max }-p\right)$, i.e. identical to the NaSch model with randomization $p$. For large densities $1-\rho \ll 1$, on the other hand, the flow is given by $J(\rho) \approx\left(1-p_{0}\right)(1-\rho)$ which corresponds to the NaSch model with randomization $p_{0}$. For densities close to $\rho=1$, only cars with velocities $v_{j}=0$ or $v_{j}=1$ exist. The number of moving cars goes to zero so that asymptotically the flow is completely determined by $p_{0}$.

The microscopic structure of the jammed states in the VDR model differs from those found in the NaSch model. While jammed states in the NaSch model contain clusters with an exponential size-distribution [23], one can find phase separation in the VDR model. The reason for this behaviour is the reduction of the outflow from a jam. If the outflow from a jam is maximal, any small jam in the free flow regime dissolves immediately since the outflow from such a jam is larger than the global flow. Therefore phase separation can not occur in that case. However, if the outflow from a jam is reduced, the density in the free flow regime is smaller than the density of maximum flow and cars can propagate freely in the low density part of the lattice. Due to the reduction of the density in the free flow regime, no spontaneous formation of jams is observable in the stationary state, if fluctuations in the free flow regime are rare.

This picture is supported by a simple phenomenological approach. Obviously the flow in the homogeneous branch is given by $J_{\text {hom }}=\rho\left(v_{\max }-p\right)=\rho v_{f}$, because every car can move with the free-flow velocity $v_{f}$. Assuming that the high density states are phase separated, we can obtain the second branch of the fundamental diagram. The phase separated states consist of a large jam and a free flow regime, where each car moves with velocity $v_{f}$. The density in the free flow regime $\rho_{f}$ is determined by the average waiting time $T_{w}=\frac{1}{1-p_{0}}$ of the first car in the jam and $v_{f}$, because neglecting interactions between cars, the average distance of two consecutive cars is given by $\Delta x=T_{w} v_{f}+1=\rho_{f}^{-1}$. Using the normalisation $L=N_{J}+N_{F} \Delta x\left(N_{F(J)}\right.$ is the number of cars in the free flow regime (jam)) we find that the flow is given by

$$
J_{\text {sep }}(\rho)=\left(1-p_{0}\right)(1-\rho) .
$$

Obviously $\rho_{f}$ is precisely the lower branching density $\rho_{1}$, because for densities below $\rho_{f}$ the jam-length is zero. It should be noted that this approach is only valid for $p_{0} \gg p$ and $v_{\max }>1$. The condition $p \ll 1$ guarantees that interactions of cars due to velocity fluctuations are rare. As a consequence, the jam is compact in that limit. For increasing $p$, the jam becomes less dense. In the case $v_{\max }=1$, cars can stop spontaneously, even in the free-flow regime. If $p_{0}$ is large enough, these cars might initiate a jam. This is the basic reason why hysteresis is usually not observed for $v_{\max }=1$.

Measurements of the average flow show that the lower branch of the fundamental diagram is not stable near the density $\rho_{1}$, if small system sizes are considered. Therefore we performed a more detailed stability analysis of the homogeneous and the jammed 

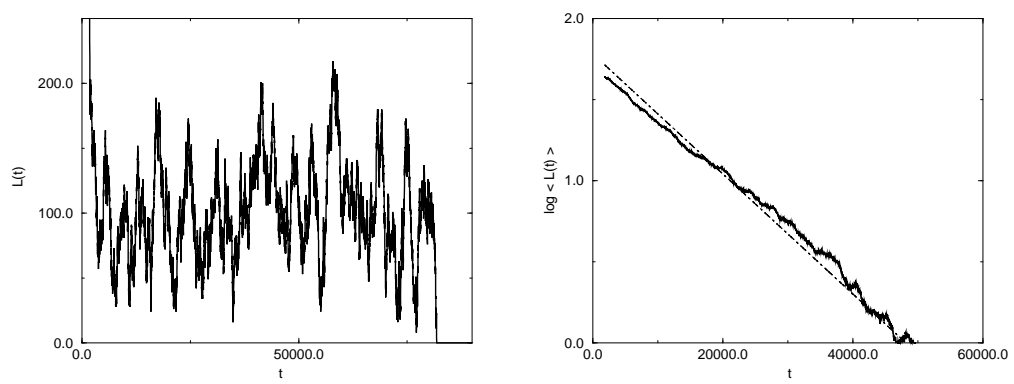

Figure 5: Time-dependence of the length $L_{J a m}(t)$ of the jam for $\rho=0.095$. The left part of the figure shows the time evolution of the length $L_{J a m}(t)$ of one sample. The average $\left\langle L_{J a m}(t)\right\rangle$ over 10000 samples (right part of the figure) shows an exponential decay.

state near $\rho_{1}$ and $\rho_{2}$. Close to $\rho_{1}$, the large jam present in the initial configuration dissolves and the average length $\left\langle L_{J a m}(t)\right\rangle$ decays exponentially in time (Fig. 5). It should be noted that this behaviour is not the consequence of a continuous "melting" of the large jam. In contrast, the jam-length $L_{J a m}(t)$ is strongly fluctuating without any obvious systematic time-dependence (Fig. 5). Once a homogeneous state without a jammed car is reached, no new jams are formed. Therefore the homogeneous state is stable near $\rho_{1}$. For large system sizes the jammed states are stable for $\rho \geq \rho_{1}$, also for densities only slightly above $\rho_{1}$, because the average length of the jam is proportional to the system size, while the fluctuations grow sub-extensive.

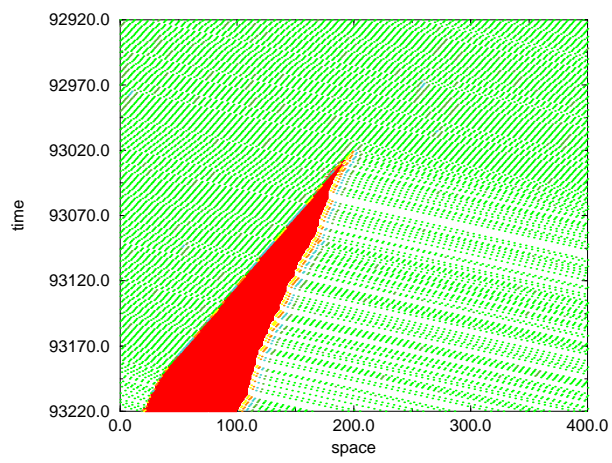

Figure 6: Space-time diagram of the VDR model for $\rho=0.15, L=400, p=0.01$ and $p_{0}=0.5$. The homogeneous initial state is not destroyed immediately, but after approximately 93000 lattice updates. In the outflow regime of the jam the density is reduced compared to the average density. 
Analogous to the metastable jammed states near $\rho_{1}$, homogeneous initializations for densities slightly above $\rho_{2}$ lead to metastable homogeneous states with short lifetimes. Fig. 6 shows the spontaneous formation of jams due to velocity fluctuations. The finite lifetimes of the homogeneous states are the qualitative difference between this model and the cruise-control limit [24] of the NaSch model, where the time evolution of homogeneous states at low densities is completely deterministic.

For large system sizes the density difference $\Delta \rho=\rho_{2}-\rho_{1}$ decreases. This can be explained by looking at the mechanism of an emerging jam. Jams emerge due to velocity fluctuations in dense regions of the homogeneous states, where the distance $d_{j}+1$ between consecutive cars is less than $v_{\max }$. In these regions all following cars have to slow down if a car in front breaks in the randomization step. Overreactions of following cars finally can cause jams. The probability to find clusters of an appropriate length for a given density is proportional to the system size. Therefore we expect that all homogeneous states are unstable for $\rho>\rho_{1}$ in the thermodynamic limit.

For higher values of $p$ the lifetime of homogeneous states at densities $\rho>\rho_{1}$ is very small. Nevertheless one can observe the same microscopic structure of the high density states as long as the outflow of a jam is sufficiently reduced. A rough estimate for the minimal difference between $p_{0}$ and $p$ necessary to observe phase separation can be obtained from the following arguments based on the features of a typical fundamental diagram of the VDR model at small $p$ and large $v_{\max }$ (see Fig. (7). In the following we assume that the fundamental diagram is perfectly linear
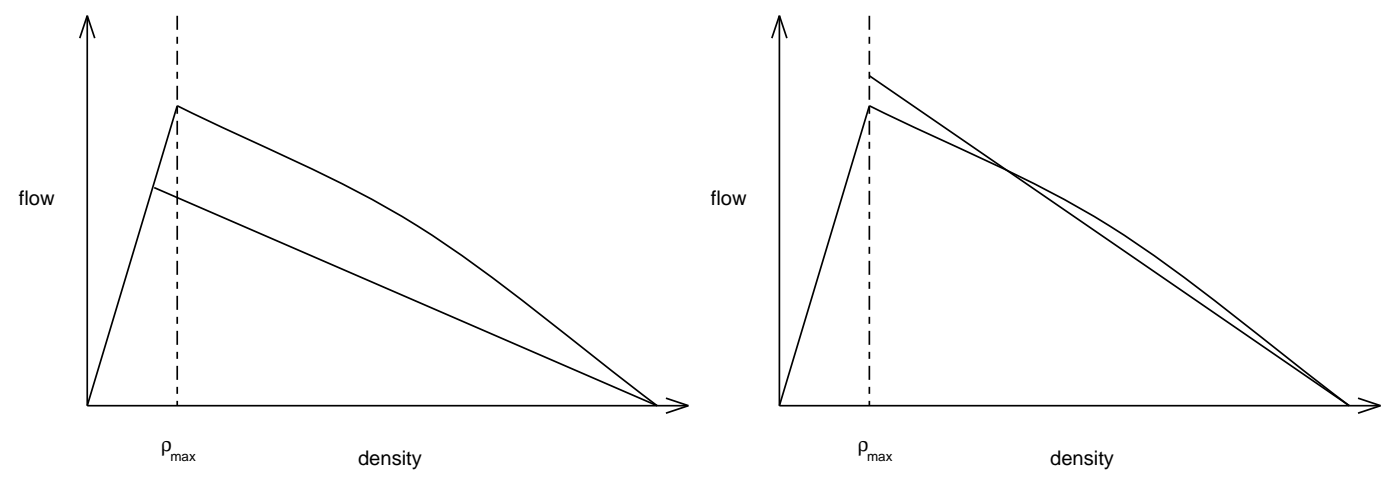

Figure 7: Illustration of the flow branches a) in the presence of phase separation and b) without phase separation.

up to $\rho_{2}$, i.e. $J_{\text {hom }}(\rho)=\rho\left(v_{\max }-p\right)$. As mentioned before, the phase-separated branch is described by $J_{\text {sep }}(\rho)=\left(1-p_{0}\right)(1-\rho)$ (see Eq. (2)). In order to find phase separation, the flow in the phase-separated branch at density $\rho_{2}$ must be lower than the flow in the homogeneous branch, i.e. $J_{\text {hom }}\left(\rho_{2}\right)>J_{\text {sep }}\left(\rho_{2}\right)$ (left part of Fig. (7). For a situation as depicted in the right part of Fig. 目 (i.e. for $J_{\text {hom }}\left(\rho_{2}\right)<J_{s e p}\left(\rho_{2}\right)$ ), no phase-separation would be found. In the following we approximate $\rho_{2}$ by $\rho_{\max }$, 
the maximum of the corresponding NaSch model with randomization $p$. Using the estimate $\rho_{\max }=(1-p) /\left(v_{\max }+1\right)$ [26], the inequality for the current at $\rho_{2} \approx \rho_{\max }$ can be used to obtain an estimate for the minimal difference $p_{0}-p$ necessary to observe phase separation (see Fig. 8). Near the deterministic limits $(p=0$ and $p=1)$ and for large values of $v_{\text {max }}$, already a small difference $p_{0}-p$ suffices to generate phase separation. For all nondeterministic cases the states of maximum flow are not completely homogeneous and the flow in the high density branch is somewhat lower than $J_{\text {sep }}(\rho)=\left(1-p_{0}\right)(1-\rho)$ for larger values of $p$. In addition, the large jam is not compact anymore.

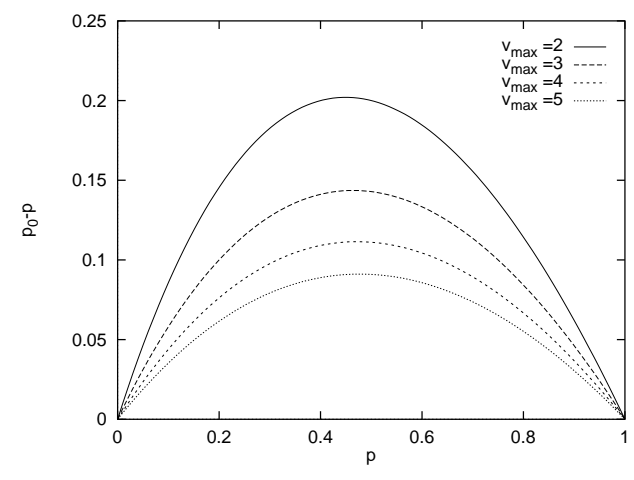

Figure 8: Estimates for the minimum difference $p_{0}-p$ necessary to observe phase separation.

In order to substantiate the picture developed in this section we also investigated correlation functions and the effect of perturbations (i.e. stopping a car temporarily to induce a jam) [27]. The results are in full agreement with our interpretation and will be presented elsewhere [28].

Another interesting result concerns the outflow from a jam. Our simulations show [28] that the outflow is (almost) independent of the density of cars and depends only on $p$. This in agreement with measurements on real traffic [17] where it was found that the outflow only depends on road and weather conditions and typical characteristics of the cars. Furthermore we have found that a structure consisting of two separated jams is rather stable and can exist for long times. This is also in agreement with experimental observations [17].

For $v_{\max }=1$ we used the so-called car-oriented mean-field theory [29] in order to obtain an analytical description of the fundamental diagram. The results are in good agreement with simulations [27]. As expected, one finds no phase separation at any $p_{0}$ for $p>0$. In the deterministic limit, however, a hysteresis curve can be observed for $p_{0}>0$. The fundamental diagram constists of two linear branches and is in perfect agreement with simulations [28]. 


\section{Summary and Discussion}

The ability to describe the existence of hysteresis is a serious test for any traffic flow model. In this paper we have investigated several variants of the NaSch model and showed numerically the existence of metastable states in a density region close to the maximum flow.

The hysteresis in the fundamental diagram is related to the existence of metastable states. The latter are a consequence of phase separation in a certain density regime. The reason for this phase separation, on the other hand, is a reduced outflow from jams which destabilizes clusters forming in the outflow region. Such a reduction can be incorporated most easily by introducing slow-to-start rules which try to model the restart behaviour of standing vehicles more realistically. The three models presented here used different s2s rules which can be classified as spatial, temporal and velocitydependent, respectively. For maximum velocity $v_{\max }>1$ the models exhibit a similar behaviour (see below). For $v_{\max }=1$ the spatial s2s rule of the T2 model is exceptional since it leads to a existence of a phase transition to a completely jammed state in the limit $p_{t} \rightarrow 1$ where $p_{t}$ is the s2s parameter.

The NaSch model with velocity-dependent randomization shows the coexistence of phase separated and homogeneous states in a density interval near the density $\rho_{2}$ of maximum flow. Near $\rho_{2}$ interactions between cars become important and one can find spontaneous formation of jams. Therefore the reduction of the density in the outflow regime of a jam leads to stable phase separated states. The reduction of interactions between cars in the free flow regime can be confirmed by a phenomenological approach, which gives very accurate results. In contrast to the cruise-control limit of the NaSch model [24], where also metastable states can be found, fluctuations are present in both coexisting states.

The results presented here have interesting applications. In real traffic one is usually interested in stabilizing the homogeneous branch of the fundamental diagram in order to maximize the throughput. This can be done using signals to control the inflow, as in the case of the Lincoln tunnel in New York [30]. Results of simulations corresponding to such a situation will be presented in a future publication [28].

In [22] a family of space-continuous models has been investigated. Depending on the values of the maximum acceleration and deceleration three classes can be distinguished. Class III shows no realistic behaviour since no spontaneous jams are formed. A continuous analogue of the NaSch model belongs to class II (high decelaration limit). Here one finds spontaneous formation of jams, but no metastable states. Finally, in class I spontaneous jamming as well as metastable states are found. On a macroscopic level, models in classes I and II can be distinguished by the ordering of the densities $\rho_{f}$, the density of the outflow from a jam, and $\rho_{c}$, where the density of a homogeneous flow becomes unstablef. The discrete models discussed here have

\footnotetext{
${ }^{4}$ Often it is not easy to define these quantities properly, especially in the presence of noise.
} 
very simple braking rules, but nevertheless exhibit the behaviour of class III. The existence of metastable states is therefore not related to the use of continuous space coordinates or "realistic" braking rules [8, 9] (e.g. anticipation of the behaviour of the driver ahead).

Already the NaSch model has a tendency towards the formation of metastable states. The outflow from a megajam is maximal only in the deterministic case $p=0$ and in the so-called cruise-control limit [24]. In the generic case $p>0$ the outflow is not maximal [31], but organizes itself towards the 'critical' current $J_{c}$ where the correlation length becomes maximal [26]. Since the difference between $J_{\max }$ and $J_{c}$ is rather small, it is very difficult to observe metastable states in the NaSch model.

The considerations in this paper show the flexibility of the CA approach to traffic flow problems. A rather simple and natural extension of the rules of the NaSch model allows us to describe the formation of metastable states in the fundamental diagram. The introduction of a velocity-dependent randomization $p(v)$ makes it possible to control the properties of the free flow and congested flow independently. Experimentally it has been found [17] that for real traffic the reduction of the outflow $J_{\text {out }}$ compared to the maximum flow $J_{\max }$ is approximately $J_{\text {max }} / J_{\text {out }} \approx 1.5$. This value can be used to determine a realistic value of $p_{0}-p$.

If one is willing to give up an overly realistic description of the interactions between the vehicles one can obtain rather simple CA models capable of describing even the fine-structure of traffic flow in a satisfactory way.

\section{References}

[1] K. Nagel, M. Schreckenberg: J. Physique I2, 2221 (1992)

[2] D.E. Wolf, M. Schreckenberg, A. Bachem (Eds.): Traffic and Granular Flow, World Scientific (1996)

[3] M. Schreckenberg, D.E. Wolf (Eds.): Traffic and Granular Flow '97, Springer (1998)

[4] D. Helbing: Verkehrsdynamik, Springer (1997)

[5] A.D May: Traffic Flow Fundamentals, Prentice Hall (1990)

[6] M. Rickert, K. Nagel: Int. J. Mod. Phys. C8, 483 (1997)

K. Nagel, C.L. Barrett: Int. J. Mod. Phys. C8, 505 (1997)

[7] J. Esser, M. Schreckenberg: Int. J. Mod. Phys. C8, 1025 (1997)

[8] S. Krauß, P. Wagner, C. Gawron: Phys. Rev. E54, 3707 (1996)

[9] S. Krauß, P. Wagner, C. Gawron: Phys. Rev. E55, 5597 (1997) 
[10] B. Kerner, S.L. Klenov, P. Konhäuser: Phys. Rev. 56, 4200 (1997)

[11] M. Bando, K. Hasebe, A. Nakayama, A. Shibata, Y. Sugiyama: Phys. Rev. E51, 1035 (1995)

[12] S. Yukawa, M. Kikuchi: J. Phys. Soc. Jpn. 64, 35 (1995)

[13] D. Helbing: Phys. Rev. 53, 2366 (1996)

[14] J. Treiterer: Ohio State Technical Report No. PB 246094 (1975)

[15] B.S. Kerner, H. Rehborn: Phys. Rev. Lett. 79, 4030 (1997)

[16] D. Helbing: Phys. Rev. E55, R25 (1997)

[17] B.S. Kerner, H. Rehborn: Phys. Rev. E53, R1297 (1996)

[18] A. Schadschneider, M. Schreckenberg: Ann. Physik 6, 541 (1997)

[19] M. Takayasu, H. Takayasu: Fractals 1, 860 (1993)

[20] M. Fukui, Y. Ishibashi: J. Phys. Soc. Jpn. 66, 385 (1997)

[21] S.C. Benjamin, N.F. Johnson, P.M. Hui: J. Phys. A29, 3119 (1996)

[22] S. Krauß: in [3]

[23] A. Schadschneider: in [3]

[24] K. Nagel, M. Paczuski: Phys. Rev. E51, 2909 (1995)

[25] R. Barlovic, L. Santen, A. Schadschneider, M. Schreckenberg: in [3]

[26] B. Eisenblätter, L. Santen, A. Schadschneider, M. Schreckenberg: Phys. Rev. E57, 1309 (1998)

[27] R. Barlovic: Diploma Thesis, Universität Duisburg (1998)

[28] R. Barlovic, L. Santen, A. Schadschneider, M. Schreckenberg: in preparation

[29] A. Schadschneider, M. Schreckenberg: J. Phys. A30, L69 (1997)

[30] H. Greenberg, A. Daou: Operations Res. 8, 524 (1960)

[31] B. Eisenblätter: Diploma Thesis, Universität Duisburg (1996) 